https://dx.doi.org/10.4314/jpb.v18i3.8

Vol. 18 no. 3, pp. 237-244 (September 2021)

http://ajol.info/index.php/jpb
Journal of

PHARMACY AND

BIORESOURCES

\title{
Use of Rasch Wright map to understand the quality of Healthcare Workers' Knowledge, Attitudes, and Practices for Uncomplicated Malaria (HKAPIUM)
}

\author{
Nanloh S. JIMAM ${ }^{*}$, Nahlah E. ISMAIL ${ }^{2}$, Dauda A. DANGIWA ${ }^{1}$, Maxwell L.P. DAPAR ${ }^{1}$, \\ Comfort N. SARIEM ${ }^{1}$, Lomak A. PAUL ${ }^{1}$, Suwaiba G. MOHAMMED ${ }^{1}$, Danlami W. \\ DAYOM ${ }^{1}$
}

${ }^{1}$ Department of Clinical Pharmacy and Pharmacy Practice, Faculty of Pharmaceutical Sciences, University of Jos, Jos. Nigeria. $\quad{ }^{2}$ Department of Clinical Pharmacy, Faculty of Pharmacy, MAHSA University, Selangor, Malaysia.

Received $8^{\text {th } J u n e ~ 2021 ; ~ A c c e p t e d ~} 3^{\text {rd }}$ August 2021

\begin{abstract}
The present study assessed the dimensionality and item difficulty targeting to person ability of HKAPIUM scale using Rasch Wright map approach. A HKAPIUM instrument (Cronbach's alpha =0.71) containing eighteen (18)-items was administered to 121 trained healthcare professionals involved in the management of uncomplicated malaria in 24 selected primary health care (PHC) facilities in Plateau state, Nigeria. The respondents filled and returned the instrument and the data was analyzed using Bond\&Fox software ${ }^{\circledR}$. The Rasch principal components analysis (RaschPCA) of the item residuals indicated variance explained values of 52 (knowledge), 41.1 (attitudes), and 55.4 (practices) and eigenvalues of the first contrast as 1.2, 1.8, and 1.5, respectively, which were within acceptable values that indicated the unidimensionality of the three constructs of the HKAPIUM scale. The result showed moderate items difficulty levels for all the three constructs. The mean person ability levels of respondents with-respect-to knowledgerelated items were low, while their mean person ability levels in endorsing the attitudes and practices-related items were good. The study revealed unidimensionality of the 3 constructs of HKAPIUM scale, with observed mismatch between item difficulty levels of the constructs with most of the person ability levels of the respondents.
\end{abstract}

Keywords: Healthcare professionals; HKAPIUM scale; Rasch-PCA; Unidimensionality.

\section{INTRODUCTION}

Healthcare professionals' knowledge, attitudes and practices instrument for uncomplicated malaria (HKAPIUM) is a selfadministered scale designed and validated using both the classical test theory (CTT) and Rasch measurement model approaches for assessing healthcare professionals' knowledge, attitudes and practices on the management of uncomplicated malaria in primary healthcare (PHC) facilities $[1,2]$. The use of CTT had enabled the classification of the HKAPIUM-related items into primary dimensions, in addition to its validity and Cronbach's alpha reliability [2]. Similarly, items fitness to model and item/person separation/reliability indices had been investigated through Rasch analysis [1], thus the need to further explore other characteristics

*Correspondence. E-mail: jimamnanloh@gmail.com Tel: +234-8020318993.

ISSN 0189-8442

(cc) BY-Nc 2021. Published by Faculty of Pharmaceutical Sciences, University of Jos, Nigeria. Under Creative Commons Attribution-Non-Commercial 4.0 International License. https://creativecommons.org/licenses/by-nc/4.0/ 
of the scale to increase its effectiveness and acceptability using Rasch analysis.

The Rasch model which is regarded as a unidimensional measurement model have added advantage in test measurement over the CTT approach based on its fundamental mathematical principles that respondents were more likely to endorse easy items than the difficult ones, and all items in an instrument were more likely to be correctly endorsed by respondents with high ability on the constructs compared to those with low ability levels [35]. According to Apple and Neff [3] and Boone and Noltemeyer [6], this principle which was based on a dichotomous right and wrong test notion, has also become very useful in the analysis of other types of data, including ordinal data (example, Likert scale data) in health and social sciences. In addition, it is a useful instrument for determination of the unidimensionality of an ordinal scale like Likert scale type data using principal component analysis (PCA) of items residuals, items and persons' fitness to model, items/person separation and reliability of an instrument, and its ability to detect and appropriately locate items according to their levels of difficulties and person ability levels using item-person map (Wright map) [3, 4, 7]. The fitness and item/person separation/reliability indices had been earlier reported $[1,2]$. This present study aimed to confirm the unidimensionality and targeting of the item difficulty to person ability using Wright map.

\section{METHODS}

Data Collection: Approval for the conduct of the study was granted by the Joint Research Review and Ethics Committee, Research Management Centre (RMC), MAHSA University, Malaysia (Ref. number: RMC/EC01/2016; Dated 25/11/2016). This letter was used to obtain permission from Plateau State Ministry of Health, Jos, Nigeria, and the directors of PHCs of the selected LGAs were appropriately communicated and informed consent obtained before data collection

The study was a cross-sectional survey conducted in 24 selected primary healthcare (PHC) facilities of Plateau state, Nigeria. The purposive sampling method was used to recruit 121 healthcare professionals involved in the management of uncomplicated malaria to participate in the study. They were selfadministered eighteen (18)-items' HKAPIUM instrument (Cronbach's alpha $=0.71$ ) developed for assessment of healthcare professionals' knowledge, attitudes, and practices (KAP) on uncomplicated malaria, who appropriately filled and returned same for statistical analysis.

Statistical analysis: The filled and returned instruments were checked by the researcher for completeness, after which the data was manually entered into statistical package for social sciences (SPSS) version 23 according to coding formats of 'no' $=0$, 'not sure' $=0$, and 'yes' $=1$, for responses to knowledge-related items, while the attitudes and practices-related responses were respectively coded as 5, 4, 3, 2, and 1 for 'strongly agree' and 'very often', 'agree' and 'often', 'neutral' and 'sometimes', 'disagree' and 'rarely', and 'strongly disagree' and 'never'.

Principal component analysis (PCA) of the item residuals was calculated for each of the constructs separately to determine their unidimensionalities using the Rasch measurement model through Bond\&Fox software $^{\circledR}$. A construct was considered unidimensional when the variance explained by the measure was $\geq 40 \%$, and the eigenvalues for the first contrast of the residual was $<2[5,8,9]$.

To further understand the strengths and weaknesses of the validated instruments, the raw scores obtained from the respondents' responses to the study instruments were mathematically transformed into logarithms by Rasch analysis, and the item and persons' 
responses were placed on the same logit scale called item-person map. This is also known as Wright map (named after Benjamin Wright) based on the item difficulty and person ability levels $[4,5,8]$. Although, in theory, the logits scale values range from negative to positive infinity [3, 8], Apple and Neff [3] had reported that extension from -5 to +5 has been more feasible in practice. Based on the model prediction, there should be even distributions of item difficulty levels in accordance to the person ability levels, and the presence of such optimal targeting of item difficulty to person ability would indicate good quality of the constructs and the instrument for the assessment of healthcare professionals' KAP $[4,7]$.

\section{RESULTS}

Dimensionalities of the constructs. Table 1 shows that the three constructs (knowledge, attitudes, and practices) of the HKAPIUM scale each have a single dimension because the variance explained values for the measures were $>40 \%$ and all the eigenvalues of the first contrast were $<2$.

\section{Wright Map Indicating Item Difficulty and Person Ability Levels Distributions. Figure} 1 shows the item difficulty levels of the knowledge-related items presented on the right hand-side of the vertical line of the Wright map arranged from less difficult (item 2 at the bottom) to the most difficult ones (item 8 on top) based on the logit scale values. Similarly, the person ability levels of the respondents were presented on the left side of the map and the model used ' $p$ ' to indicate each of the 121 healthcare professionals, and their distributions from bottom to top was an indication of their ability levels in responding to the items. Those located on top of the map were regarded as having high ability in responding to the knowledge-related items, and those arranged in the middle of the map were categorized as having the average ability, while those at the bottom were persons with low ability. Twenty-four (24) respondents located on +1 logit were considered the healthcare professionals that had the highest ability in correctly responding to the entire items of the knowledge-related instrument during the study. This was followed by 17 respondents whose ability levels were located below - 1 logit, but above the mean person ability level of -1.87 (denoted by ' $M$ ' on the vertical line of the Wright map), although, the items were considered difficult for them to correctly endorse. The majority (80) of the respondents' ability levels was below the mean ability levels. In general, considering the person ability levels of the respondents and item difficulty level of the knowledge construct's items, there was less divergence of the item difficulty level distribution around the mean (M) item difficulty, contrary to the person ability level distribution from person ability mean (M).

The item difficulty level for attitudes construct were also presented on the right-hand side of the Wright map and the locations of the items on the map were based on the difficulty levels. All the 5 items were distributed between -1 and +1 logits of the map around its item difficulty mean (M) of zero (0), with item 1 been the easiest while item 3 was the most difficult. Furthermore, healthcare professionals' ability level based on their responses to the attitude-related items were presented on the left side of the vertical line of the Wright map and the distribution cut across from the bottom of the map to the top (Figure 2). Fifty-seven (57) of the respondents were located at the top of the map (between +4 and +5 logits) and were considered as having the highest ability to respond to the attitudesrelated items, while 58 of them were located between +1 and +4 logits which were all around the mean (M) ability scale of 2.97 which indicated their moderate ability to correctly answer the attitudes-related items. Only 6 persons spread between -1 and +1 logit below the person ability mean (M). 
The Wright map in Figure 3 also showed the item difficulty level for practicesrelated items which were distributed between -1 and +1 logits of the map around its mean (M) item difficulty levels of zero (0), with item 1 been the easiest followed by item 3 , while item 2 was the most difficult. Furthermore, the ability levels of 70 of the respondents were located between +4 and +5 logits, while 42 were located around the mean (M) ability scale of +2.10 logits which indicated their high and moderate abilities levels, respectively, based on their responses to the practices-related items. Only 9 persons were located between 2 and 0 logit below the mean ability levels, indicating those with poor ability. Although, almost all the respondents considered the items as been easy for them to endorse because of their ability levels, the uneven distribution of the items across the average person ability region of the map (Figure 3), might suggest poor targeting of item difficulty to the patients' ability by the instrument.

Table 1: Explained and Unexplained Variance for HKAPIUM constructs $(\mathrm{N}=121)$

\begin{tabular}{llllllll}
\hline Constructs & $\begin{array}{l}\text { Variance } \\
\text { explained } \\
\text { eigenvalue }\end{array}$ & $\begin{array}{l}\text { Variance } \\
\text { explained } \\
(\%)\end{array}$ & $\begin{array}{l}\text { Unexplained } \\
\text { variance } \\
\text { eigenvalue }\end{array}$ & $\begin{array}{l}\text { Unexplained } \\
\text { variance } \\
(\%)\end{array}$ & $\begin{array}{l}\text { First } \\
\text { contrast } \\
\text { eigen } \\
\text { value }\end{array}$ & $\begin{array}{l}\text { First } \\
\text { contrast } \\
\text { explained } \\
\text { variance } \\
(\%)\end{array}$ & $\begin{array}{l}\text { Disattenuated } \\
\text { person } \\
\text { measure } \\
\text { correlation }\end{array}$ \\
\hline Knowledge & 10.9 & 52.0 & 10.0 & 48.0 & 1.2 & 10.6 & \\
Attitudes & 2.8 & 41.1 & 5.0 & 58.9 & 1.8 & 22.5 & \\
Practices & 3.7 & 55.4 & 3.0 & 44.6 & 1.5 & 22.3 & \\
\hline
\end{tabular}

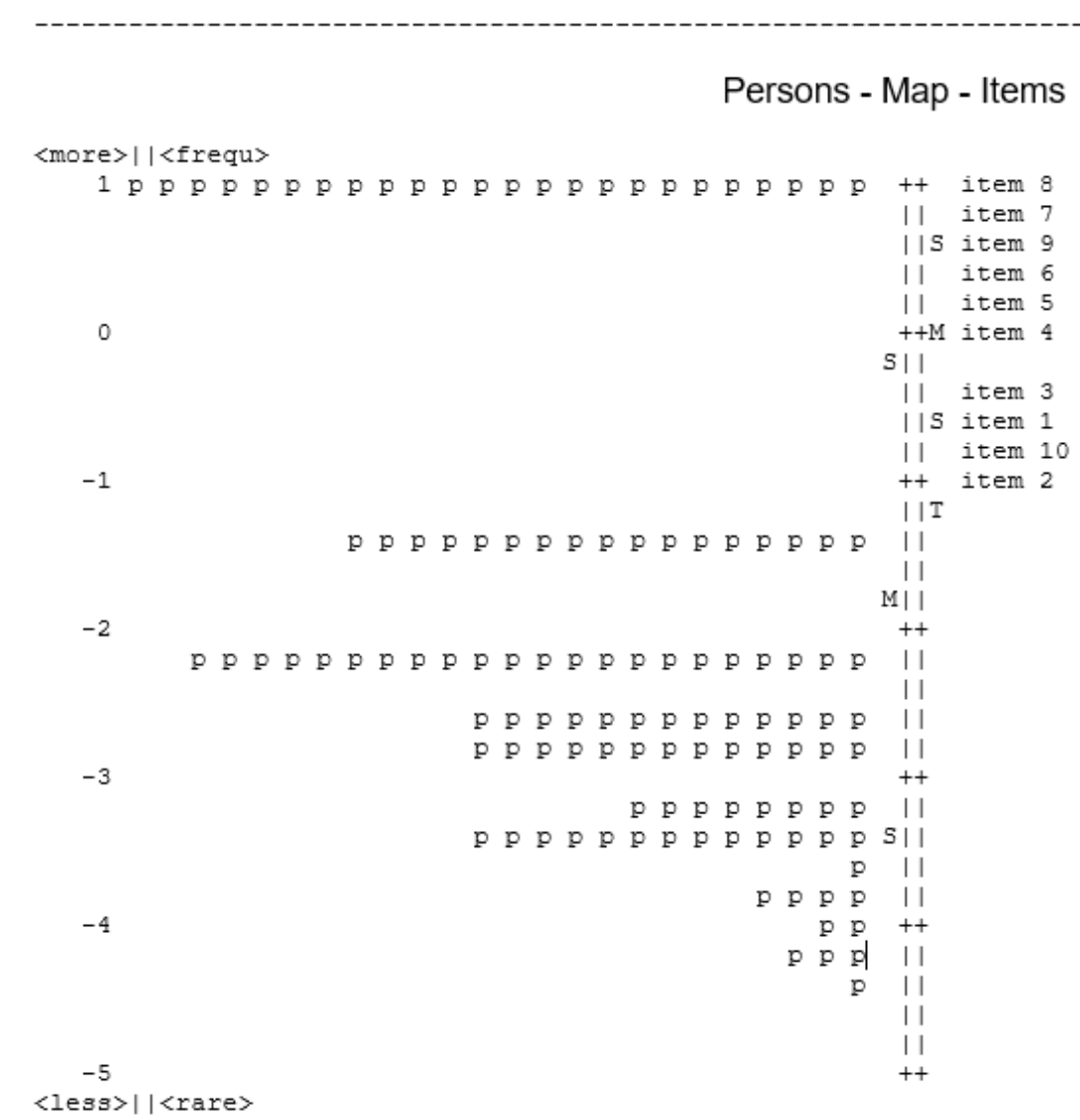

Figure 1: Wright map of person ability and items difficulty for knowledge construct of HKAPIUM (N = 121) 


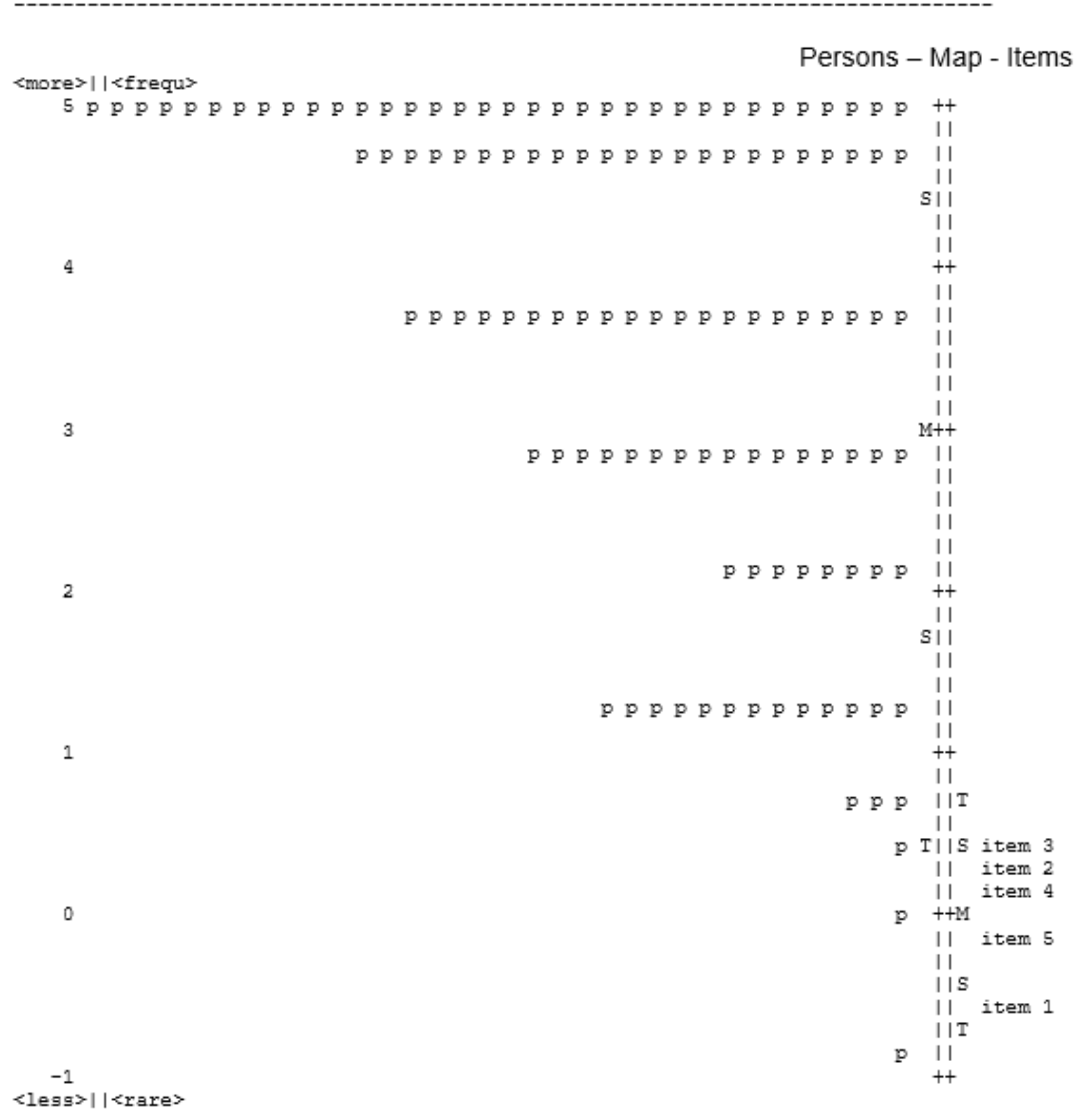

Figure 2: Wright map of person ability and items difficulty for attitudes construct of HKAPIUM (N = 121) 


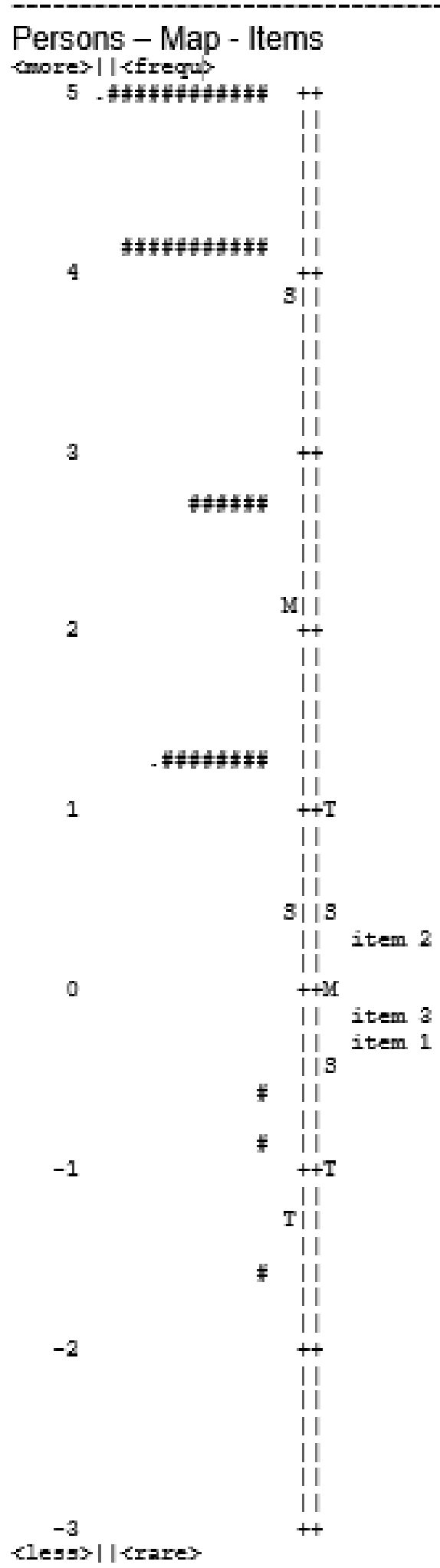

Figure 3: Wright map of person ability and items difficulty for practices construct of HKAPIUM (N = 121). Each '\#' represents 3 persons and each '.' represents 1 person. 


\section{DISCUSSION}

The present study reported the dimensionalities and item difficulty and person abilities of the three constructs of the HKAPIUM instrument. It has been reported that an ideal instrument should contain items with difficulty levels spread evenly along with the person ability levels such that the gap in their mean logits should be narrow or the same, indicating optimal targeting of the item difficulty to person ability, which might be a good signal to the quality of the instrument [3, $5,7]$.

The variance explained values for all the three constructs (knowledge, attitudes and practices) were above the minimum acceptable value of $40 \%$, and the respective eigenvalues of the first construct of 1.2 (knowledge), 1.8 (attitudes), and 1.5 (practices), which were all $<2$, indicating the unidimensionality of the constructs [5]. The clustered healthcare professionals' knowledge, attitudes, and practices items around the mean item difficulty level value of \pm 1 signified moderate difficulty levels for all the items. Furthermore, the mean person ability levels falling on the negative side of the vertical axis of the item-person maps (-1.87 logits) for knowledge construct indicated low ability levels of the majority of the respondents

incorrectly answering the knowledge items, as the items were considered difficult for them, and such distribution patterns indicated poor targeting of the item among persons with moderate and low abilities as there was no alignment between the items difficulty level with the person ability levels $[5,7]$. On the contrary, the mean ability levels of the respondents in answering the attitudes and practices-related items were all positive and located +2.97 logits (attitudes constructs) and +2.10 logits (practices constructs) which indicated good ability levels of the respondents in endorsing the items as they consider them as easy, but there were mismatched between the item difficulty with high and moderate abilities respondents in answering the attitudes-related items. Similar poor targeting was observed between item difficulties with abilities of respondents who were located in the high ability quartile of the map [7].

The observed poor targeting of items difficulty levels with person ability levels of the respondents might be linked to the earlier reported low items and persons separation and reliability of the three constructs reported as $>$ $1<2$ (item and person separation) and reliability in the range of $0.61-0.75$, except for knowledge construct whose separation and reliability index values were, respectively, 2.99 and 0.9 [1].This is because it had been reported that the strength of an instrument to correctly detect and separate respondents into low and high abilities depends on its person separation and reliability index values, and separation index values of $>2$ (and reliability $>0.8$ ) indicates a better likelihood of the instrument to correctly detect and separate the respondents into the number of strata base on their ability levels in the sample, while lower values $(<2$, and person reliability $<0.8)$ indicates less likelihood of the instrument to correctly differentiate the ability levels of the respondents [3, 5]. On a similar note, the strength of the instrument to correctly detect and classify items base on their difficulty levels also depends on the items separation index values, with values $>3$ indicating the instruments' strength of been more likely to correctly detect and differentiate the items difficulty levels than those with the value of < 3.

Conclusion. Each of the three constructs (knowledge, attitudes, and practices) that constituted the HKAPIUM instrument were shown to be single dimensional constructs. The Wright map showed moderate items difficulty levels for all the knowledge, attitudes, and practices-related items for HKAPIUM scale, with majority of the respondents possessing low ability levels for knowledge items, and moderate to high ability levels for attitudes and 
practices-related items. The overall distribution patterns of the item difficulty levels with-respect-to the healthcare professionals' ability levels were not satisfactory as there was poor targeting of item difficulty levels across some of the respondents' ability levels.

\section{REFERENCES}

1. Ismail NE, Jimam NS, Dapar MLP, Ahmad S. Validation and Reliability of Healthcare Workers' Knowledge, Attitude, and Practice Instrument for Uncomplicated Malaria by Rasch Measurement Model. Frontiers in Pharmacology, 2020; 10:1521. Doi: 10.3389/fphar.2019.01521

2. Ismail NE, Jimam NS, Dapar MLP. Assessment of primary health care workers' knowledge, attitudes and practices on uncomplicated malaria management in Plateau state, Nigeria. International Journal of Pharmaceutical Science and Research, 2019; 10(1): 286-293. Doi: 10.13040/IJPSR.09758232.10(1).286-93.

3. Apple M, Neff P. Using Rasch measurement to validate the Big Five factor marker questionnaire for a Japanese university population. Journal of Applied Measurement, 2012; 13(3): 276-96.
4. Boone WJ, Staver JR, Yale MS. Rasch analysis in the human sciences, Dordrecht, Netherlands: Springer, 2014.

5. Linacre JM. A user's guide to Winsteps (user's manual and software), 2012. [Online], from: http://www.winsteps.com/winsteps.htm [accessed on 20 March, 2018].

6. Boone WJ, Noltemeyer A. Educational psychology and counselling | review article: Rasch analysis: A primer for school psychology researchers and practitioners. Cogent Education 2017; 4:1. Doi: 10.1080/2331186X.2017.1416898

7. Abdullah N, Noranee S, Khamis MR. The use of Rasch wright map in assessing conceptual understanding of electricity. Pertanika Journal of Social Sciences \& Humanities, 2017; 25 (S): 81-92.

8. Bond TG, Fox CM. Applying the Rasch model: Fundamental measurement in the human sciences, third edition. New York: Routledge, 2015.

9. Rasch G. Weblogs models for some intelligence and Student tests. Chicago: The University of Chicago Press, 1980. [Online], from: www.uchicago.edu/ucp/books/chicago [accessed on $28^{\text {th }}$ November, 2017]. 